\title{
Aspectos morfológicos de la enfermedad hepática inducida por drogas
}

\section{Morphological Issues of Drug Induced Liver Disease}

Rocío del Pilar López Panqueva, MD. ${ }^{1}$

1 Médica Patóloga Hospital Universitario Fundación Santa Fe de Bogotá. Universidad de Los Andes. Bogotá, Colombia

Fecha recibido: $13-11-14$

Fecha aceptado: 24-11-14

\begin{abstract}
Resumen
La enfermedad hepática inducida por drogas es un fenómeno multifacético y su espectro morfológico es muy variado imitando cualquier patrón de daño hepático, tanto en pacientes expuestos en forma aguda o crónica, en aquellos susceptibles en forma idiosincrática a una dosis terapéutica o por toxicidad intrínseca, a su vez puede estar afectada por otros factores como son los genéticos, la edad o el sexo, el estado nutricional, la exposición a otros fármacos o la existencia de una enfermedad de base; puede ser la única manifestación clínica del efecto adverso de una droga o estar acompañado de manifestaciones sistémicas o de otros órganos, e incluso puede llegar a ser fatal (1).

Su incidencia no está bien definida, algunos estudios afirman que la incidencia global es variable encontrándose entre 1-15 x 100.000 personas/año, en USA ocurren 20 nuevos casos x 100.000 habitantes/año. Se han descrito como causantes de lesión hepática más de 900 drogas, productos herbales, homeopáticos, suplementos dietéticos o toxinas, sean productos naturales o de la industria farmacéutica, utilizados o no en dosis terapéuticas, que son las responsables de aproximadamente $15 \%$ de consultas y hospitalizaciones por ictericia, hepatitis aguda o crónica; en la población adulta, por encima de los 50 años, llega a $40 \%$ de todos los casos de hepatitis. Es también la causante de 11-50\% de casos de falla hepática aguda. Los datos publicados indican que los antibióticos son responsables entre un $27-46 \%$ de los casos, seguidos por medicamentos para enfermedades del sistema nervioso central entre 13-17\%, antiinflamatorios y analgésicos de $5-17 \%$ y los productos herbales $9 \%$. Nuevos biomarcadores y el uso de microRNA se están estudiando y serán prometedores en un futuro cercano para identificar pacientes que puedan presentar hepatotoxicidad inducida por medicamentos.

Son tantos los tipos de lesión hepática atribuidos a estos agentes que solo podremos dar algunos ejemplos en este artículo, basados en los patrones de daño hepático y enfatizando la importancia de una adecuada y profunda correlación clínica $(2,3)$.
\end{abstract}

\section{Palabras clave}

Hígado, biopsia, toxicidad, drogas, tóxicos, fármacos, patrones, necroinflamatorio, colestasis, esteatosis, esteatohepatitis, té verde, acetaminofén, esteroides, amiodarona, metimazol, nitrofurantoína, clorpromacina, anticonceptivos orales, metrotrexate.

\section{GENERALIDADES}

El hígado se convierte en un blanco susceptible de daño por ser el primer órgano en contacto con agentes ingeridos, el sitio más importante de concentración de xenobióticos y el lugar donde ocurre la mayor biotransformación. La suscep- tibilidad a presentar toxicidad está modificada por numerosos factores, es más frecuente en adultos que en niños y las mujeres son más susceptibles a presentar toxicidad crónica. Algunos medicamentos producen lesión dependiendo de la dosis o del tiempo de exposición, otras por idiosincrasia metabólica o interacción con otros medicamentos. 


\begin{abstract}
Drug-induced liver disease is a multifaceted phenomenon which has a varied morphological spectrum that mimics other patterns of liver damage both in cases of acute drug exposure and in cases of chronic exposure to drugs. Those patients who are idiosyncratically susceptible at the therapeutic dose or to intrinsic toxicity may also be affected by other factors including genetic factors, age, sex, nutritional status, exposure to other drugs and the existence of an underlying disease. The only clinical manifestation of the disease may be the adverse effect of a drug, but it can also be accompanied by systemic manifestations and manifestations in other organs, and it can even be fatal (1).

The incidence of drug-induced liver disease is not well defined, but some studies claim that its overall annual incidence varies between $1 / 100,000$ people and 15/100,000 people. In the United States, twenty new cases per 100,000 inhabitants occur every year. More than 900 natural and pharmaceutical drugs, herbal medicines, homeopathic products, dietary supplements and toxins have been reported to cause liver damage. This can occur whether or not they are used at normal therapeutic doses. These cases are responsible for about $15 \%$ of consultations and hospitalizations for jaundice, acute hepatitis, and chronic hepatitis in adults above the age of 50 , and in up to $40 \%$ of all cases of hepatitis. Drug-induced liver disease also accounts for $11 \%$ to $50 \%$ of all cases of acute liver failure. Published data indicate that antibiotics are responsible for between $27 \%$ and $46 \%$ of cases, that drugs for diseases of the central nervous system are responsible for between $13 \%$ and $17 \%$, anti-inflammatory and analgesic agents are responsible for between $5 \%$ and $17 \%$, and herbal products are responsible for $9 \%$. New biomarkers and the use of microRNA are being studied and may become promising alternatives in the near future for identifying patients susceptible to drug-induced hepatotoxicity.

There are so many types of liver damage attributed to these agents that only give some examples can be provided in this article. These examples have been chosen on the basis ofn the patterns of liver damage with emphasis on the importance of proper and thorough clinical correlation $(2,3)$.
\end{abstract}

\title{
Keywords
}

Liver biopsy, toxicity, drugs, toxins, patterns, necroinflammatory, cholestasis, steatosis, steatohepatitis, green tea, acetaminophen, steroids, amiodarone, methimazole, nitrofurantoin, chlorpromazine, oral contraceptives, methotrexate.

Frecuentemente, al suspender la medicación la lesión se resuelve, aun cuando puede tomar varias semanas o meses y es muy raro que persista a pesar de suspenderla (4).

El diagnóstico de la injuria hepática inducida por cualquier fármaco, toxina, suplemento dietético, vitamina o producto herbal u homeopático, suele ser un desafío tanto para el clínico como para el patólogo, no hay hallazgos clínicos ni de laboratorio específicos. El "Council for International Organizations of Medical Sciences" (CIOMS) clasifica estas lesiones como de patrón hepatocelular, colestásico o mixto; pueden simular cualquiera de las enfermedades hepáticas primarias agudas y crónicas y así mismo pueden encontrarse todos los patrones morfológicos conocidos como son necroinflamatorio, esteatosis, esteatohepatitis, colestásicos, vasculares, tumorales, cirrosis, entre muchos otros. Estos patrones de daño hepático reflejan el mecanismo fisiopatológico que indujo el daño hepático como por ejemplo hipersensibilidad, trastorno autoinmune $o$ metabólico, y además pueden sugerir su etiología (5).

Es un diagnóstico de exclusión donde en algunas ocasiones la biopsia hepática puede contribuir, un adecuado criterio clínico y el conocimiento sobre el potencial agente lesivo hepático llevan a la aproximación diagnóstica correcta.
Aunque la biopsia hepática sigue siendo considerada como el estándar de oro para el diagnóstico de hepatotoxicidad inducida por fármacos, esta no es un procedimiento usado en la práctica médica rutinaria, es requerida usualmente como una prueba diagnóstica cuando el cuadro clínico no es claro o hay discordancia con las pruebas de laboratorio, o para determinar el patrón de daño hepático, puede ser importante para excluir agentes tóxicos, esclarecer mecanismos de injuria, o evaluar el grado de severidad o su cronicidad, también cuando no existe la sospecha clínica y se dificulta el diagnóstico diferencial $(6,7)$.

En este artículo estudiaremos las formas más frecuentes de presentación según los patrones morfológicos observados en cada una de ellas; en el intento de permitir un enfoque práctico en el diagnóstico de la enfermedad hepática inducida por drogas o toxinas. En las diferentes tablas se enumeran solo algunos de los agentes usualmente implicados en cada uno de los patrones que se describirán.

\section{TIPO AGUDO}

Es una de las formas más frecuentes de presentación, ocurre en $90 \%$ de los casos, cuando la exposición ha sido 
menor a 3 meses y muestra varios espectros de lesión hepática $(4-6,8,9)$ :

1. Patrón de daño hepatocelular, citotóxico o citolítico, caracterizada por daño del hepatocito y que se traduce en necrosis hepatocelular la cual puede ser zonal o masiva, asociada o no a esteatosis. Clínicamente los síntomas semejan una hepatitis aguda viral con ictericia hepatocelular, elevación de las aminotransferasas (8 a 100 veces su valor normal), con fosfatasa alcalina normal o solo ligeramente evada. En este grupo de pacientes hay tendencia a presentar falla hepática fulminante. El diagnóstico diferencial incluye hepatitis viral, o por cualquier microorganismo, hepatitis autoinmune y la enfermedad de Wison (tabla 1).

2. Patrón de esteatosis aguda macro o microvesicular, o semejando esteatohepatitis no alcohólica; presentan ictericia leve con niveles de aminotransferasas menores que los vistos cuando existe necrosis hepatocelular; en algunos casos como cuando se acompaña de insuficiencia hepática, acidosis láctica con ausencia o mínima alteración de las aminotransferasas, compromiso sistémico severo y pronóstico pobre; la lesión hepática es causada por la alteración del metabolismo graso que inhibe la función mitocondrial, dependiendo del agente los síntomas inician en promedio entre los 7 y 28 días posteriores. Debemos incluir en el diagnóstico diferencial las esteatohepatitis de etiología metabólica por alcohol, obesidad o diabetes mellitus, la enfermedad de Wilson y la hepatitis viral $\mathrm{C}$, si la esteatosis es de vacuola pequeña recordar excluir entidades como síndrome de Reye o el hígado agudo graso del embarazo (tabla 2).

3. Patrón colestásico semeja clínicamente una obstrucción de la vía biliar extrahepática, aun cuando también se deben tener en mente que se han descartado procesos sépticos, falla cardíaca, hepatitis virales. Se manifiesta con ictericia de tipo obstructivo y prurito, puede acompañarse de dolor abdominal y se encuentran parámetros bioquímicos que apoyan colestasis con fosfatasa alcalina y gammaglutamil transferasa elevadas, puede existir hiperbilirrubinemia y los niveles de aminotransferasas rara vez superan 5 veces su valor normal.

4. Patrón mixto: colestásico y hepatocelular, combina manifestaciones clínicas y patológicas de los dos patrones, es uno de los más frecuentes y característicos dado que rara vez se produce un patrón semejante en enfermedades agudas hepáticas de otra etiología. Las manifestaciones clínicas simulan una hepatitis aguda acompañada de prurito y fatiga, las cifras de fosfatasa alcalina (FA), aminotransferasas tanto ALT como AST están elevadas en proporción semejante y la relación ALT/ FA está entre 2 y 5 , definiendo el patrón mixto (tabla 3 ).

Tabla 1. Patrón de daño hepatocelular agudo.

\begin{tabular}{|c|c|}
\hline Tipo de lesión & Medicamento \\
\hline $\begin{array}{l}\text { Necrosis con } \\
\text { balonización } \\
\text { panacinar }\end{array}$ & $\begin{array}{l}\text { Ácido para-amino-salicílico (PAS), halotano, } \\
\text { indometacina, isoniazida, metildopa, } \\
\text { nitrofurantoína, sulfonamidas, té verde, hoja } \\
\text { de chaparral (creosote bush, Larrea tridentata) }\end{array}$ \\
\hline $\begin{array}{l}\text { Hepatitis } \\
\text { mononucleosis-like" }\end{array}$ & Dapsona, fenitoína, PAS, sulfonamidas, \\
\hline $\begin{array}{l}\text { Necrosis submasiva } \\
\text { pericentral }\end{array}$ & $\begin{array}{l}\text { Acetaminofén, envenenamiento por amanita } \\
\text { phalloides, cobre, halotano, ketoconazol, } \\
\text { propiltiouracilo, tricloroetileno y tetracloride } \\
\text { de carbón. Germander (Teucrium genus), } \\
\text { Pennyroyal (Mentha pulegium, Hedeoma } \\
\text { pulegioides) }\end{array}$ \\
\hline $\begin{array}{l}\text { Necrosis submasiva } \\
\text { zona } 1\end{array}$ & $\begin{array}{l}\text { Toxicidad por sulfato ferroso y } \\
\text { envenenamiento por fósforo }\end{array}$ \\
\hline Necrosis masiva & $\begin{array}{l}\text { Fenitoína, halotano, isoniazida, Kava (Piper } \\
\text { methysticum) }\end{array}$ \\
\hline
\end{tabular}

Tabla 2. Patrón esteatósico agudo.

\begin{tabular}{|c|c|}
\hline Tipo de lesión & Medicamento \\
\hline $\begin{array}{l}\text { Esteatosis } \\
\text { microvesicular }\end{array}$ & $\begin{array}{l}\text { Ácido acetilsalicílico, alcohol, aflatoxina, } \\
\text { amiodarona, ácido valproico, calcio, cocaína, } \\
\text { piroxicam, tetraciclina (administración IV) }\end{array}$ \\
\hline $\begin{array}{l}\text { Esteatosis } \\
\text { macrovesicular }\end{array}$ & $\begin{array}{l}\text { Alcohol, asparginasa, antiinflamatorios no } \\
\text { esteroideos, corticoesteroides, didanosina, } \\
\text { envenenamiento por fósforo, linezolid, } \\
\text { metrotrexate, metoprolol, mercurio, minoxiclina, } \\
\text { nutrición parenteral, nifedipino, agentes } \\
\text { quimioterapéuticos, tetraciclina }\end{array}$ \\
\hline Esteatohepatitis & $\begin{array}{l}\text { Alcohol, amiodarona, agentes quimioterapéuticos, } \\
\text { didanosine, estrógenos sintéticos, nifedipino, } \\
\text { metotrexate, tamoxifeno }\end{array}$ \\
\hline
\end{tabular}

Tabla 3. Patrón colestásico y mixto, agudo.

\begin{tabular}{ll}
\hline \multicolumn{1}{c}{ Tipo de lesión } & \multicolumn{1}{c}{ Medicamento } \\
\hline $\begin{array}{l}\text { Colestasis con daño } \\
\text { ductal o colangitis aguda } \\
\text { Colestasis pura o blanda }\end{array}$ & $\begin{array}{l}\text { Alopurinol, carbamazepina, hidralazina, } \\
\text { paracuat } \\
\text { Anticonceptivos orales, azatioprina, } \\
\text { anabólicos, esteroides, estrógenos, } \\
\text { metimazol, mercaptopurina } \\
\text { Hepatocelular y } \\
\text { colestásico }\end{array}$ \\
& $\begin{array}{l}\text { Captopril, cumadin, eritromicina, } \\
\text { etambutol, fenotiazinas, fenilbutazona, } \\
\text { griseofulvina, isoniazida, metimazol, } \\
\text { nutrición parenteral total, tiazidas, } \\
\text { verapamilo }\end{array}$ \\
\hline
\end{tabular}




\section{Hallazgos histopatológicos}

\section{Patrón de daño hepatocelular agudo $(4,6,7)$}

- El compromiso hepatocelular con necrosis y degeneración balonizante comprometiendo todo el acino (panacinar) con mínima colestasis o ausencia de esta, semejando clínicamente una hepatitis aguda de etiología viral, con lesión difusa del parénquima e inflamación lobulillar con linfocitos, macrófagos, células plasmáticas, usualmente es rica en eosinófilos, variable en su localización acinar o portal, en algunos puede observarse inflamación portal mínima y apoptosis, desarreglo lobulillar con fragmentación de la trama reticular, formaciones rosetoides de hepatocitos, acompañados de cambios regenerativos con binucleación, mitosis y trabéculas hepatocitarias gruesas .

- Una variante asociada con linfocitosis sinusoidal y marcada activación de las células de Kupffer semejando una hepatitis por mononucleosis (seudomononucleosis o mononucleosis - "like"), con necrosis focal y actividad.

- Necrosis submasiva en zona pericentral (zona 3), la necrosis se acompaña de inflamación variable y colapso de la trama reticular, hiperplasia de células de Kupffer llenas de lipofuschina y leve colestasis.

- Necrosis submasiva en zona 1 (periportal) con necrosis de coagulación y mínima respuesta inflamatoria, o acompañada de acúmulo de grasa de vacuola grande.

- Necrosis masiva, dando un patrón de daño como el observado en las hepatitis agudas virales fulminantes.

\section{Patrón esteatósico agudo $(10,11)$}

- Esteatosis de microvacuola o vacuola pequeña que semeja al hígado graso agudo del embarazo o a la enfermedad de Reye, pequeñas gotas grasas ocupando el citoplasma de los hepatocitos, con núcleo central hipercromático.

- Esteatosis de macrovacuola donde hay reemplazo citoplasmático por una gran vacuola grasa con desplazamiento periférico del núcleo.

- Esteatohepatitis con esteatosis usualmente de vacuola mediana o grande, lesión hepatocelular con necrosis o balonización e inflamación, con o sin cuerpos de Mallory-Denk.

\section{Patrón colestásico agudo (4, 7, 9, 12)}

- Se observa depósito de bilis en hepatocitos y/o canalículos, con estasis biliar, inflamación portal, proliferación colangiolar o lesiones de los conductos biliares y conductillos.

- La colestasis pura o blanda se caracteriza por manifestarse con marcada ictericia, coluria y prurito en un paciente que por lo demás manifiesta sentirse muy bien. No hay alteración de la fosfatasa alcalina ni de las aminotransferasas o existe mínima elevación de estas siempre por debajo de dos veces su valor normal.

\section{Patrón mixto colestásico y necroinflamatorio $(4,7,9)$}

En la biopsia hepática se observa marcada necrosis de hepatocitos, células apoptóticas o necrosis confluente e inflamación acompañada de estasis biliar, colestasis hepatocanalicular y proliferación de colangiolos.

\section{TIPO CRÓNICO}

La progresión a cronicidad ocurre cuando la exposición es mayor a 6 meses con persistencia de injuria bioquímica y se ha descrito entre $11 \%$ y $13 \%$ de los casos ya que muchos de los fármacos o tóxicos que describimos anteriormente, como causantes de daño hepático agudo, también pueden causar lesión crónica, semejando tanto en la clínica como en sus cambios histopatológicos una hepatitis crónica, algunos incluso llevan a fibrosis importante y cirrosis (4-8).

1. Patrón necroinflamatorio crónico es indistinguible tanto en las manifestaciones clínicas como en los hallazgos histopatológicos de una hepatitis crónica, especialmente de tipo autoinmune, siempre descartando por estudios serológicos las hepatitis virales. Hay reportes de al menos 24 productos relacionados con esta patología, algunos de los cuales refiero en la tabla 4. Incluso se presenta más frecuentemente en mujeres, presentan signos de hipersensibilidad con rash, artralgias y eosinofilia, pueden o no existir autoanticuerpos (ANAs, ASMA, hipergammaglobulinemia), siendo extremadamente difícil el diagnóstico diferencial entre la hepatitis crónica inducida por drogas y la hepatitis autoinmune. La gravedad es variable, al retirar el medicamento se espera una resolución completa y respuesta a la terapia con corticosteroides apoya el diagnóstico (tabla 4) (13-17).

2. Las lesiones colestásicas crónicas son muy semejantes en la clínica y en su cuadro histopatológico a la cirrosis biliar primaria, colangitis esclerosante primaria o a la disminución de ductos (ductopenia). La mayoría de los casos de colestasis inducida por drogas son seguidos por una rápida mejoría de los síntomas y recuperación completa posterior a la retirada del medicamento, en algunos persiste anomalía bioquímica por varios meses, exceptuando la presencia de anticuerpos antimitocondria (AMA) que no son detectables (tabla 5).

\section{Hallazgos histopatológicos}

- Patrón necroinflamatorio crónico o hepatitis autoinmune inducida por drogas con o sin marcadores autoin- 
Tabla 4. Patrones de injuria hepática crónica.

\begin{tabular}{|c|c|}
\hline $\begin{array}{c}\text { Enfermedad hepática inducida por drogas que semeja } \\
\text { hepatitis autoinmune }\end{array}$ & Medicamento \\
\hline Hepatitis autoinmune "like" con autoanticuerpos negativos & Lisinopril, metrotrexate, sulfonamidas, tamoxifen, trazodone, uracilo \\
\hline Hepatitis autoinmune "like" ANAS (+) ASMA (+) & $\begin{array}{l}\text { Alfa-metildopa, clometacina, antiinflamatorios no esteroideos (AINES) como } \\
\text { diclofenaco, estatinas, agentes anti-TNF-a, infliximab, hidralazina, nitrofurantoína }\end{array}$ \\
\hline Hepatitis autoinmune "like" ANAs (+)ASMA (-) & Minociclina \\
\hline Hepatitis autoinmune "like" LKM (+) & Hidralazina \\
\hline Productos bien establecidos & Minociclina, nitrofurantoína, acetato de oxifenisatina, alfametil-dopa, clometacina \\
\hline Productos probables & Estatinas \\
\hline Productos biológicos & Infliximab, Adalimumab, etanercept, efalizumab, ipilimumab \\
\hline Productos menos probables & Atomoxetina, diclofenaco, dihidralazina, fenofibrato, pemolina, warfarina. \\
\hline
\end{tabular}

Tabla 5. Colestasis crónica.

\begin{tabular}{ll}
\hline \multicolumn{1}{c}{ Tipo de lesión } & \multicolumn{1}{c}{ Medicamento } \\
\hline Cirrosis biliar primaria-like & $\begin{array}{l}\text { Ajmaline, anticonceptivos orales, amitriptilina, ampicilina, barbitúricos, benoxaprofen, carbamazepina, } \\
\text { cimetidina, clorpromacina, fenitoína, haloperidol, ketoconazol, metiltestosterona, tiabendazol, tolbutamida }\end{array}$ \\
Colangitis esclerosante primaria-like & $\begin{array}{l}\text { Fluxuridine en infusión intraarterial, formalina para esterilización de los quistes por equinococo } \\
\text { Ductopenia }\end{array}$ \\
& $\begin{array}{l}\text { Aceprometacina, ajmaline, amitriptilina, amoxacilina, ampicilina, azatioprina, azitromicina, barbitúricos, clorotiazida, } \\
\text { clindamizina, diazepam, eritromicina, ibuprofeno, fenitoína, tetraciclina, tiabendazol, trimetropinsulfa }\end{array}$ \\
\hline
\end{tabular}

munes. El patrón que semeja la hepatitis autoinmune clásica muestra marcada inflamación portal y lobulillar predominantemente con plasmocitos, algunos linfocitos y eosinófilos, hepatitis de interfase y necrosis hepatocelular. En la hepatitis autoinmune inducida por drogas con marcadores autoinmunes negativos las características histológicas son indistinguibles de una hepatitis viral crónica o autoinmune, incluso con progresión a fibrosis y cirrosis (13-17).

- Patrón que semeja cirrosis biliar primaria. Los hallazgos morfológicos se caracterizan por la evidencia de colestasis crónica con cambio pseudoxantomatoso hepatocitario, acúmulo de cobre y de su proteína fijadora en hepatocitos periportales, leve a moderada inflamación portal en parches con variable pérdida de ductos. El término "vanishing" o pérdida de ductos se usa para describir los casos con marcada ductopenia.

- Patrón que semeja colangitis esclerosante primaria con esclerosis ductal de la vía biliar extrahepática o de los ductos intrahepáticos.

Otros patrones menos frecuentes pero igualmente importantes de tener en cuenta:

\section{INJURIAS VASCULARES}

Se dividen a su vez en varios cuadros morfológicos $(4,18,19)$ :
- Dilatación sinusoidal: asociada a uso prolongado de anticonceptivos orales, especialmente localizada a nivel de la zona 1. También con la azatioprina y heroína.

- Peliosis hepática, con grandes espacios llenos de hematíes sin ningún revestimiento endotelial: observada con terapia esteroidea anabólica, azatioprina, anticonceptivos orales, danazol, hipervitaminosis A y tamoxifen.

- Fleboesclerosis con el alcohol y la heroína.

- Hepatitis isquémica: amiodarona administrada por vía intravenosa.

- Enfermedad venooclusiva: por ingestión de tés que contengan alcaloides pirrolidizine, también por alcohol, exceso de vitamina A, azatioprina y dacarbazina. En pacientes con terapia inmunosupresora con ciclofosfamida, o con oxipltinum posterior a terapias por carcinoma de colon y es una complicación de terapias de quimiorradiación postrasplante de medula ósea.

- Síndrome de Budd-Chiari: ha sido reportado por uso de anticonceptivos orales, en niños que reciben nutrición parenteral total.

- Lesión de arterias y arteriolas hepáticas: algunas drogas producen una angeítis por hipersensibilidad llevando a engrosamiento de la pared, con edema endotelial, necrosis de miocitos e inflamación con eosinófilos, ocasionalmente arteritis de células gigantes en arterias portales. Se incluyen en este grupo las sulfonilureas, penicilina, fenitoína y alopurinol. 
- Trombosis e infartos relacionados con anticonceptivos orales.

- Esclerosis hepatoportal por exposición a arsénico, azatioprina o con la exposición a antiretrovirales como didadosina.

\section{REACCIONES GRANULOMATOSAS}

Varias entidades causan granulomas intrahepáticos entre ellas están procesos infecciosos, sarcoidosis, enfermedades colestásicas crónicas o sistémicas, linfomas y las drogas; se observan hasta en $15 \%$ de las biopsias hepáticas la presencia de granulomas epitelioides no caseificantes localizados en áreas portales, periportales o en el acino, algunos acompañados de necrosis focal o mínima colestasis siendo algunos ejemplos las drogas antimicrobianas como amoxacilina, cefalexina, dicloxacilina, interferón, isoniazida, nirofurantoína, quinina, penicilina, sulfonamidas; algunos anticonvulsivantes como carbamazepina, clorpromacina, diazepam, fenitoína; otros como alopurinol, amiodarona, aspirina, vacunación anti TBC, dapsona y metildopa entre muchas otras. El uso prolongado de aceites minerales para la constipación puede producir lipogranulomas. El talco, especialmente en consumidores IV de sustancias psicoactivas y también por materiales de sutura en procedimientos intrabdominales pueden llevar a granulomas en la superficie hepática $(4,7)$.

\section{FIBROSIS HEPÁTICA Y CIRROSIS}

Aun cuando la gran mayoría de las reacciones adversas a drogas se recuperan sin producir una fibrosis de importancia clínica, algunas de ellas se asocian con fibrosis periportal, pericentral o acinar.

Fibrosis en zona 1 especialmente en aquellas que han producido enfermedad necroinflamatoria crónica como las hepatitis autoinmunes-like, o las colestásicas crónicas.

Fibrosis en zona 3 ocurre a nivel subsinusoidal o pericelular por enfermedad hepática alcohólica y en hipervitaminosis A.

Hepar lobatum en el cual hay fibrosis importante formando lóbulos grandes, ha sido descrito posterior a quimioterapia en pacientes con carcinoma metastásico de glándula mamaria o de recto $(21,22)$.

La cirrosis la observamos especialmente en aquellos pacientes que han presentado necrosis hepatocelular submasiva la cual evoluciona a fibrosis, regeneración nodular y posterior cirrosis.

También se ve cirrosis establecida como complicación por terapia prolongada con metrotrexate o amiodarona. Otros ejemplos de cirrosis posterior a uso prolongado de isoniazida, iproniazidas, ácido valproico (4).

\section{NEOPLASIAS Y PSEUDOTUMORES}

En el pasado fue bien conocida la relación del uso del Thorotrast (su uso ya descontinuado) y de la terapia con arsénicos, con la presencia de angiosarcoma hepático, carcinoma hepatocelular y colangiocarcinoma. La exposición al vinil cloride con el angiosarcoma o hemangioendotelioma epitelioide $(23,24)$.

La asociación de los anticonceptivos orales y los adenomas hepáticos y la hiperplasia nodular focal está muy bien establecida y algunos casos de hepatocarcinoma. Otros agentes como los esteroides anabólicos, danazol y carbamazepina también se han asociado con adenoma hepatocelular (25) y la azatioprina ha sido relacionada con el desarrollo de hiperplasia nodular regenerativa (26).

Algunos ejemplos de los patrones anteriormente descritos son ejemplificados con las siguientes historias e imágenes.

\section{Hepatitis aguda por té verde (Camellia sinensis) (figura 1)}

Mujer de 47 años presenta ictericia de 2 meses de evolución acompañada de náusea y dolor abdominal. Desde hace 4 meses refiere estar tomando té verde para perder peso. No tiene otros antecedentes de importancia. Se encuentra elevación de las aminotransferasas 10 veces su valor normal y de la bilirrubina total a expensas de la directa, fosfatasa alcalina normal. Serologías virales y autoanticuerpos negativos.

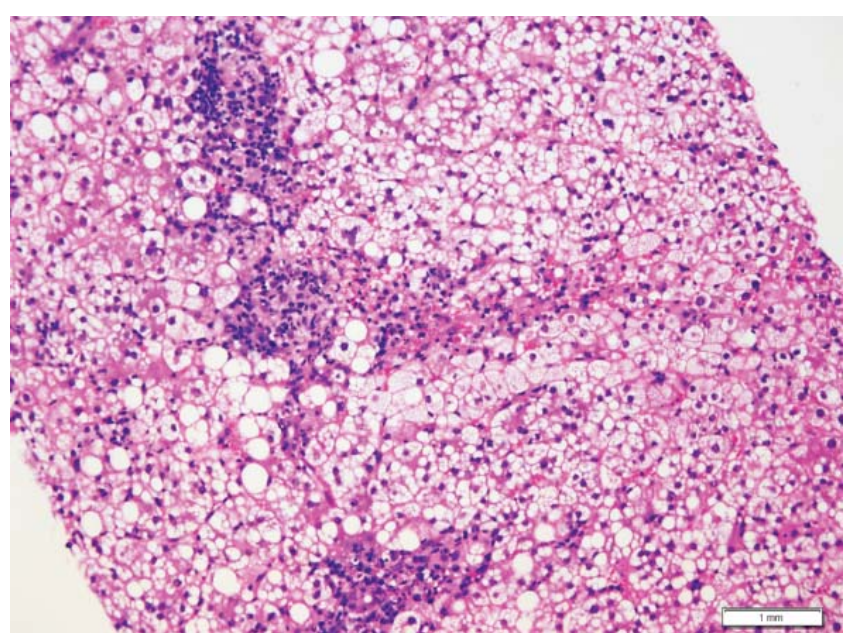

Figura 1. Hematoxilina \& Eosina 20X. Hepatitis aguda por té verde con balonización, cambio graso predominantemente microvacuolar, inflamación portal, lobulillar y hepatitis de interfase.

Realizan biopsia hepática revela edema celular o balonización, cambio graso predominantemente microvacuolar y marcada inflamación portal, lobulillar, con hepatitis de interfase y ocasionales hepatocitos apoptóticos, sin fibrosis. 
Hay numerosas descripciones de hepatotoxicidad por productos herbales y suplementos dietéticos, sea suministrada en infusiones, extractos o como componentes de estos productos; sin embargo, su incidencia es desconocida con reportes que van desde $2 \%$ hasta $50 \%$. El té verde característicamente se presenta como lesión hepática aguda e incluso falla hepática severa y muerte, aun cuando la mayoría corresponde a reportes de casos y considerados como "probable" según el score CIOMS. Existen más de 100 productos que contienen extracto de té verde, se ha demostrado que dosis menores a 1,6 gramos son bien toleradas. Su mecanismo de acción así como sus efectos adversos no están bien dilucidados, puede tener periodos de latencia que van desde 10 días hasta 7 meses posterior al inicio de su exposición. El patrón de daño más referido es el hepatocelular, aun cuando existen reportes con patrón colestásico y mixto (27).

\section{Hepatitis submasiva por acetaminofén (figura 2)}

Mujer de 25 años, obesa, toma 30 tabletas de $500 \mathrm{mg}$ cada una de acetaminofén, acompañadas de alcohol en altas dosis, en un intento de suicidio. Al siguiente día de su ingesta presenta numerosos episodios de náusea y vómito por lo cual es llevada a urgencias. Ingresa taquicárdica, hipotensa, afebril, confusa y con asterixis. Encuentran ictericia, con bilirrubina total en $15,5 \mathrm{mg} / \mathrm{dl}$, directa $14,2 \mathrm{mg} / \mathrm{dl}$ y muy marcada elevación de aminotransferasas, ALT 15,570 U/L, AST 18,733 U/L, fosfatasa alcalina $129 \mathrm{U} / \mathrm{L}$, prolongación del PT 3 veces su valor normal. Niveles de acetaminofén $141 \mathrm{mcg} / \mathrm{ml}$. Realizan trasplante hepático, en el explante hay una alteración completa de la arquitectura por desarreglo trabecular por necrosis submasiva hepatocelular con colapso de la trama reticular, presencia de células balonizadas y proliferación colangiolar con mínima inflamación.

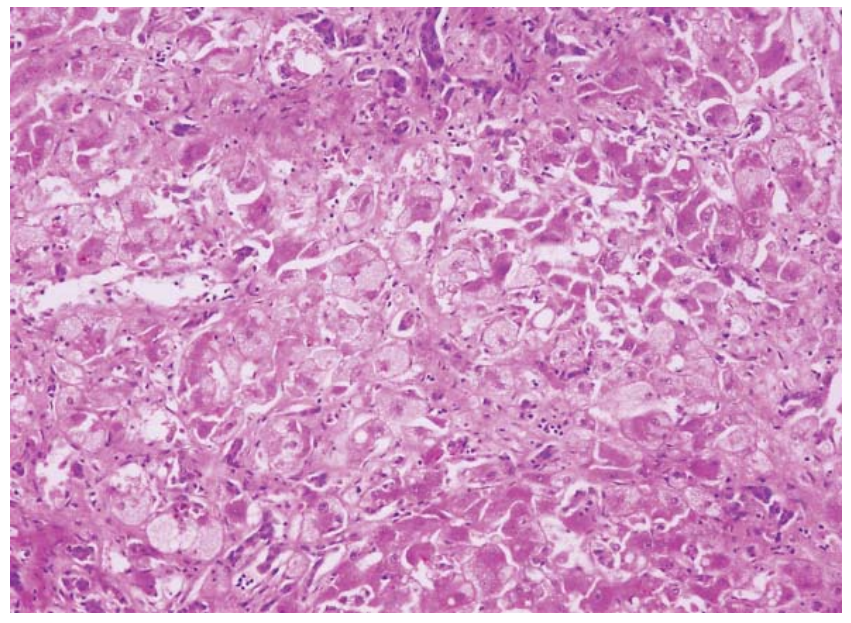

Figura 2. Hematoxilina \& Eosina 10X. Hepatitis aguda submasiva por acetaminofén. Alteración completa de la arquitectura, desarreglo trabecular con colapso, presencia de células balonizadas y proliferación colangiolar.
El daño tóxico producido por el acetaminofén es directo hepatocelular, implicado en cerca de $40 \%$ de los casos de falla hepática aguda, usualmente por sobredosis (mayor 7,5 gramos/día en adultos), los síntomas inician en las siguientes 24 a 96 horas posteriores a su ingesta llevando rápidamente a falla hepática e incluso muerte, se acompaña frecuentemente de insuficiencia renal. En forma accidental o no intencionada puede ocurrir especialmente en pacientes malnutridos, alcohólicos o con una enfermedad hepática crónica de base. A dosis tóxicas hay depleción severa del glutatión saturando todos los mecanismos de conjugación dejando libres los componentes originales que son metabolizados a compuestos intermedios muy lesivos para las células hepáticas. El sobrepeso, alcohol y medicamentos inductores del sistema citocromo P-450 pueden bajar el nivel tóxico del acetaminofén (4).

\section{Esteatohepatitis por esteroides (figura 3)}

Mujer de 30 años diagnosticada con lupus eritematoso sistémico desde los 20 años, recibe betametasona desde hace 5 años con muy buena respuesta clínica de su enfermedad de base. La dosis se ha venido disminuyendo de 6 a $1 \mathrm{mg}$ por día; sin embargo, en los controles encuentran aumento de las aminotransferasas entre 3 y 4 veces su valor normal, con aumento progresivo en el último año, al inicio de la terapia eran normales, no hay otra sintomatología asociada. Todas las demás pruebas de función hepática y serologías virales son normales. Encuentran hepatomegalia y deciden realizar biopsia hepática en la cual observamos marcada esteatosis macrovesicular panlobulillar con mínima inflamación portal con expansión fibrosa portal y subsinusoidal leve.

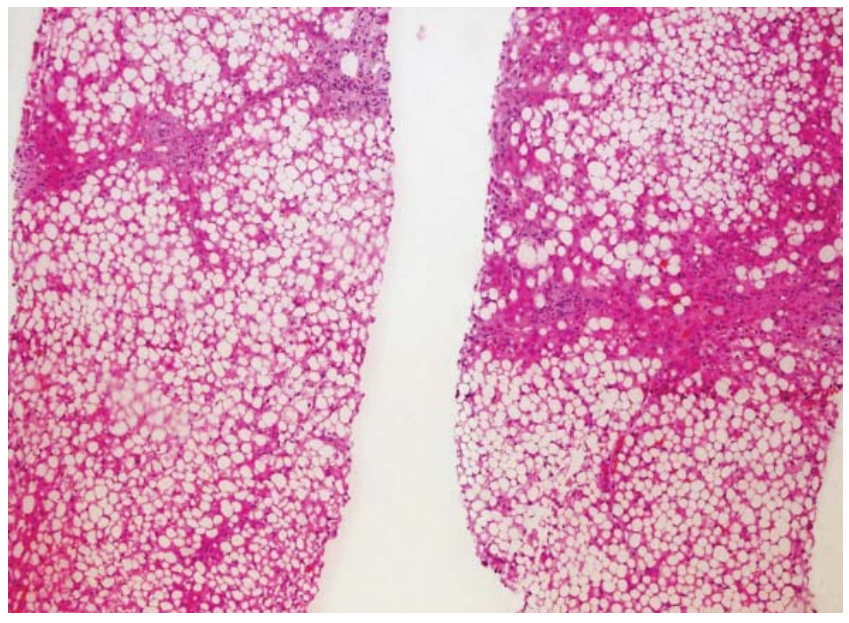

Figura 3. Esteatohepatitis por esteroides. Hematoxilina \& Eosina 10X. Esteatohepatitis por esteroides. Marcado cambio graso macrovacuolar panlobulillar con leve inflamación portal y expansión fibrosa. 
Uno de los efectos adversos del uso prolongado con glucocorticoides es la toxicidad hepática, desencadenando o empeorando una esteatosis, menos frecuentemente produce esteatohepatitis o hepatitis crónica e igualmente es poco usual la lesión hepática sintomática y progresiva. Puede ser debido al efecto mismo de los glucocorticoides sobre la resistencia a la insulina o a la alteración del metabolismo de los ácidos grasos, también puede ser el resultado del sobrepeso comúnmente presentado en los pacientes que reciben terapia con corticosteroides a largo plazo. Mientras que la esteatosis es rápidamente reversible la esteatohepatitis puede revertir muy lentamente al suspender la terapia (28).

\section{Esteatohepatitis con marcada fibrosis por amiodarona (figura 4)}

Mujer de 80 años consulta por pérdida de peso, astenia, adinamia e ictericia asociadas a dolor abdominal difuso. Antecedente de hipertensión arterial, arritmia supraventricular recibiendo metoprolol $50 \mathrm{mg} /$ día, losartan 50 $\mathrm{mg} /$ día y amiodarona $200 \mathrm{mg} /$ día en los últimos 10 años. Se encuentra hepatomegalia, leve ascitis, los estudios de laboratorio muestran bilirrubina total 9,6, bilirrubina indirecta 3,48 mg/dl, bilirrubina directa $6,12 \mathrm{mg} / \mathrm{dl}$, AST $51 \mathrm{UI} / \mathrm{L}, \mathrm{ALT} 42 \mathrm{UI} / \mathrm{L}$, fosfatasa alcalina 282, albúmina 2,03, proteínas totales 4,89 , globulina 2,86 , tiempo de protrombina $13,7 / 10,7$.

La biopsia hepática mostró marcada balonización hepatocelular con presencia de frecuentes cuerpos de Mallory en zona 1 , leve colestasis hepatocelular e inflamación, con cambio graso macrovacuolar (figura $4 \mathrm{~A}$ ) asociado a fibrosis subsinusoidal y en puentes (figura 4B).

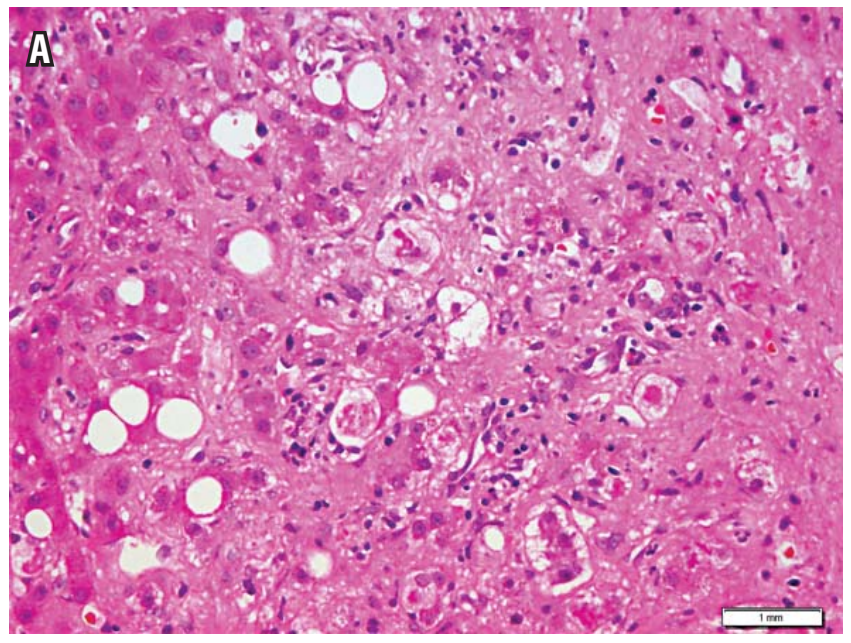

La amiodarona produce elevación de las aminotransferasas hasta en $30 \%$ de los pacientes y esteatohepatitis en $1-2 \%$ de los tratados a dosis altas y por tiempo prolongado, e incluso la dosis total acumulada persiste en el tejido aun después de suspender el tratamiento. El cuadro morfológico es muy variado observándose desde lesión hepatocelular, esteatohepatitis, colestasis, hepatitis isquémica, cirrosis o anomalías mitocondriales con lisosomas cargados de fosfolípidos (fosfolipidosis) que a la microscopia de luz pueden mostrar citoplasmas claros y de aspecto espumoso o granular en hepatocitos y células de Kupffer que en la ultraestructura corresponden a inclusiones lamelares lisosomales. También se ha asociado a raros casos de síndrome de Reye. El mecanismo de acción parece ser un daño directo de la membrana lipídica con alteración lisosomal y mitocondrial, esta última explica la presencia de esteatosis y la formación de cuerpos de Mallory con posterior fibrosis $(4,18)$.

\section{Colestasis pura por metimazol (figura 5)}

Mujer de 33 años con enfermedad de Graves, se inicia manejo con metimazol $20 \mathrm{mg} / 2$ veces por día, 3 semanas después presenta ictericia, acolia, coluria, astenia, adinamia y prurito intenso. Se encontró ictericia generalizada de piel y mucosas, sin otros hallazgos relevantes. Bilirrubina total: $17,89 \mathrm{mg} / \mathrm{dl}$, directa: $12,95 \mathrm{mg} / \mathrm{dl}$, indirecta: $4,94 \mathrm{mg} / \mathrm{dl}$, FA: 342 UI/l, GGT: 23 UI/l, AST: 82 UI/1, ALT: 93 UI/1, TP: 12/10 segundos, INR: 1,0, TPT: $25 / 23$ segundos, albúmina: 3,4 g/dl. Serologías virales, ANAs, AMA, SMA negativos. La biopsia hepática mostró únicamente marcada colestasis hepatocanalicular.

La hepatotoxicidad por metimazol es usualmente transitoria con elevación asintomática de las aminotransfera-

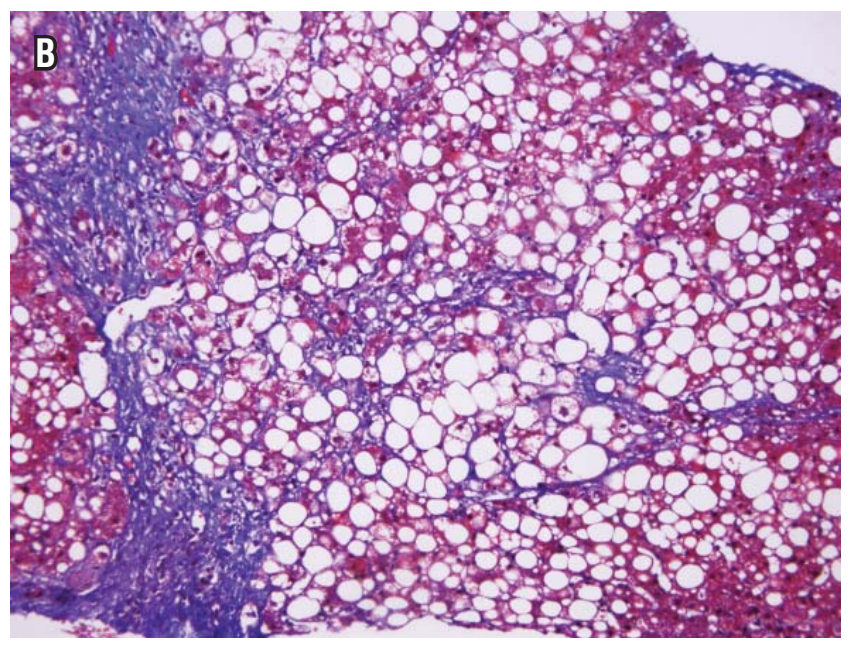

Figura 4. A. Hematoxilina \& Eosina 40X. Esteatohepatitis por amiodarona. Frecuentes células balonizadas con cuerpos de Mallory. B. Tricrómico de Masson 20X. Fibrosis portal y subsinusoidal, con marcada esteatosis. 
sas, siendo rara la hepatitis colestásica, la ductopenia o la colestasis aislada. El mecanismo de acción es desconocido; se cree que hay una reacción inmune a uno de sus productos metabólicos; cuando se presenta colestasis puede ser debida a una alteración enzimática de causa genética, por disminución del glutatión o por aumento en la concentración hepática del medicamento que produce en el hepatocito un efecto tóxico sea directo o a través de uno de sus metabolitos (12).

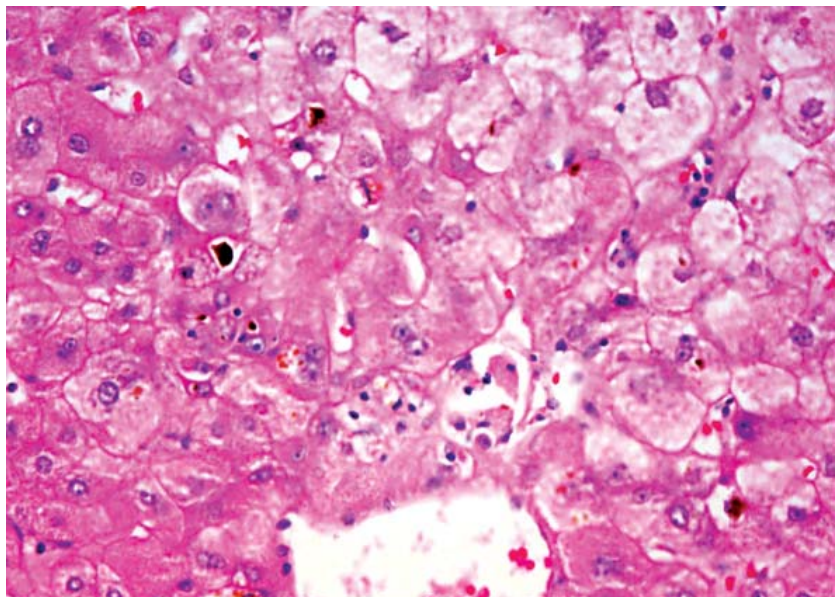

Figura 5. Hematoxilina \& Eosina 40X. Colestasis pura por metimazol. Se observa edema celular y presencia de depósito de pigmento biliar en canalículos, algunos formando pequeños tapones biliares de localización pericentral.

\section{Hepatitis autoinmune - "like” por nitrofurantoína (figura 6)}

Una mujer de 60 años ha presentado varios episodios de infección urinaria recibiendo múltiples tratamientos con antibióticos. Un mes antes nuevamente presenta sintomatología urinaria y se automedica nitrofurantoína $50 \mathrm{mg}$ / día, medicación que había recibido en ocasiones anteriores. Consulta por malestar general y náuseas, refiere que desde hace 6 meses viene presentado fatiga y cansancio. Le encuentran elevación de aminotransferasas 3-4 veces su valor normal, no hay elevación de fosfatasa alcalina y la bilirrubina es normal. Antimúsculo liso (AML) es positivo $1: 80$, pero ANAs y AMA negativos al igual que las serologías virales. Dada la persistencia de transaminitis realizan biopsia hepática encontrando inflamación portal con lesión de la placa limitante, el infiltrado es linfoplasmocitario con hepatitis lobulillar y presencia de algunos hepatocitos apoptóticos, hay expansión fibrosa portal.

Diferenciar tanto clínicamente como por hallazgos morfológicos una hepatitis autoinmune de una hepatitis "autoinmune-like" inducida por fármacos puede ser muy difícil o imposible, siendo el juicio clínico fundamental para el adecuado diagnóstico. Los antibióticos son causantes de un porcentaje importante de casos y específicamente con características que semejan una hepatitis autoinmune es de $9 \%$, siendo la nitrofurantoína y la minociclina $90 \%$ de estas, predominantemente se presenta en mujeres sin otros factores de riesgo que sugieran una clásica hepatitis autoinmune. Factores genéticos, polimorfismos y presencia de antígenos leucocitarios moduladores de la respuesta inmune, con formación de neoantígenos, activación de linfocitos T CD8 (+) y deficiencia en la regulación inmune han sido identificados como probables desencadenantes del daño hepatocitario. La suspensión del medicamento es esencial para obtener la mejoría espontánea, si hay manifestaciones clínicas y patológicas severas el uso de esteroides está indicado $(14,15)$.

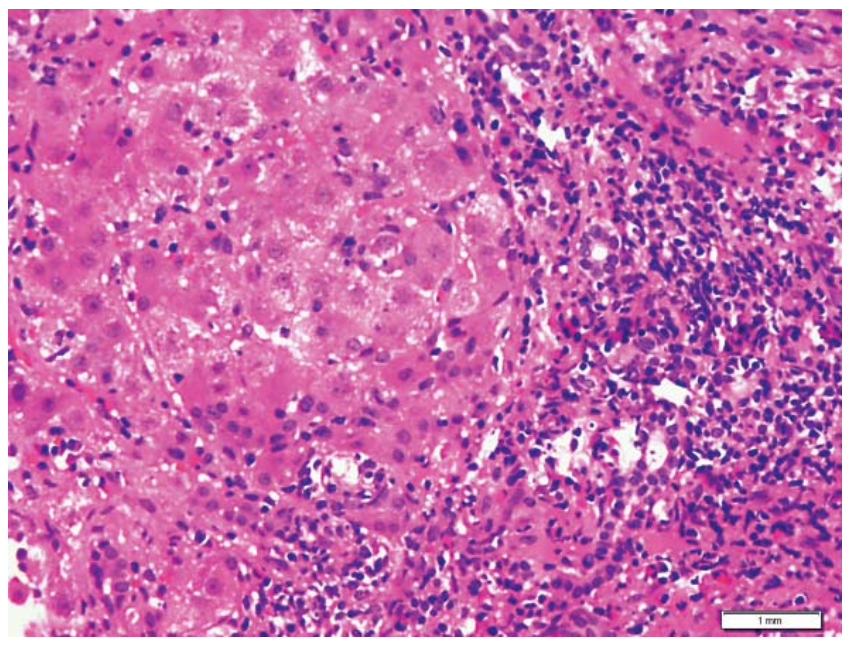

Figura 6. Hematoxilina \& Eosina $40 \mathrm{X}$. Hepatitis autoinmune-like por nitrofurantoína. Se observa expansión portal por denso infiltrado linfoplasmocitario con hepatitis de interfase y hepatitis lobulillar.

\section{Patrón colestásico crónico de tipo cirrosis biliar primaria por clorpromacina (figura 7)}

Un hombre de 55 años viene recibiendo para su depresión y desde hace 2 meses clorpromacina, inició con $50 \mathrm{mg} /$ día y actualmente $75 \mathrm{mg} /$ día. Consulta por fatiga, coluria, ictericia y prurito. Se encuentra elevación de la bilirrubina $7 \mathrm{mg} /$ dl, fosfatasa alcalina $220 \mathrm{UI} / \mathrm{L}$, leve aumento de las aminotransferasas y eosinofilia periférica. Deciden suspender el medicamento, inician antihistamínicos y colestiramina; sin embargo, persiste la sintomatología e incluso el prurito ha aumentado y 6 meses después las pruebas de función hepática duplican las cifras inicialmente observadas. Realizan biopsia hepática encontrando infiltrados portales con inflamación y cambios degenerativos ductales (figura 7A). Continúa fatiga, aumentando la ictericia, con prurito, presenta esteatorrea y pérdida de peso; dos años después, 

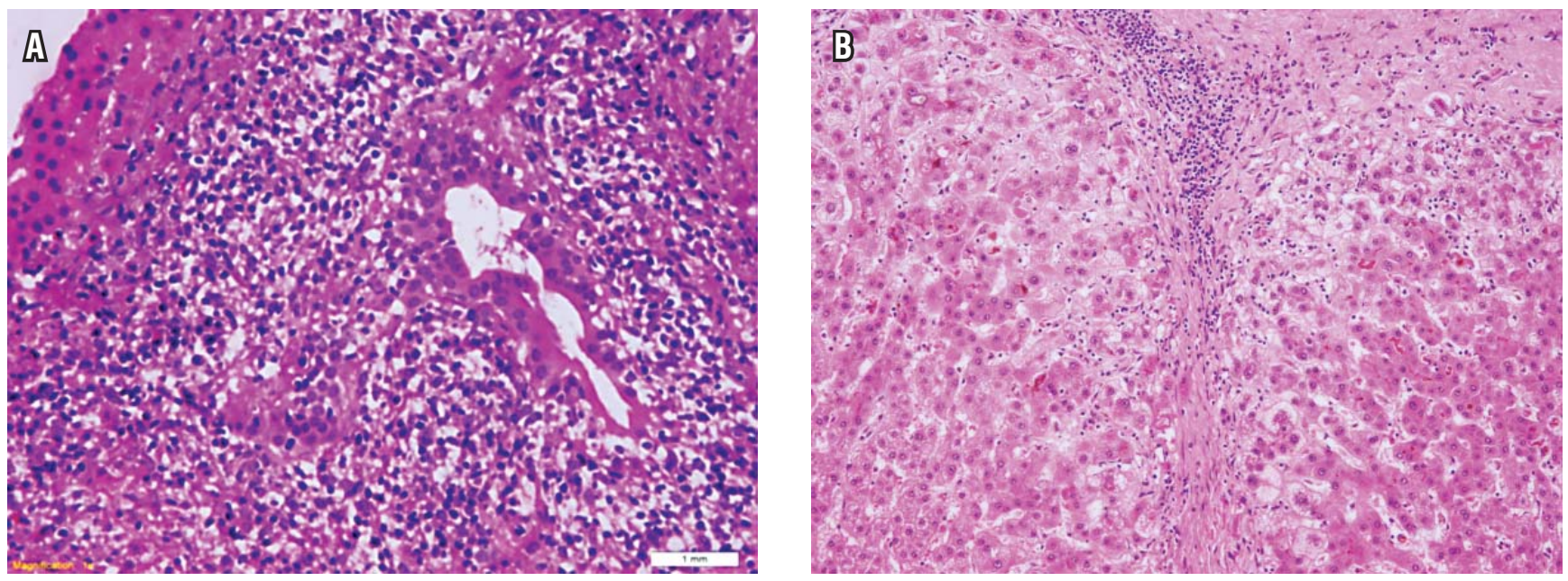

Figura 7. A. Hematoxilina \& Eosina 40X. Patrón colestásico de tipo cirrosis biliar primaria por clorpromacina. Se observa severa inflamación portal, con cambios degenerativos del epitelio ductal. B. Hematoxilina \& Eosina. Colestasis con depósito de bilis intracanalicular, leve inflamación portal, cambio xantomatoso periseptal, ductopenia de $50 \%$ y fibrosis portal y septal.

nueva biopsia muestra evidencia de colestasis crónica con leve inflamación portal, cambio xantomatoso periseptal, ductopenia de 50\% y fibrosis portal y septal (figura 7B).

Anomalías en las pruebas de función hepática han sido reportadas hasta en $40 \%$ de los pacientes en tratamiento a largo plazo con la clorpromazina, usualmente autolimitadas y revirtiendo incluso sin suspender el medicamento. Una lesión bien conocida es la colestásica tanto aguda como crónica. Manifestaciones como fiebre, rash y eosinofilia se producen en algunos de los casos. El mecanismo de acción no es conocido aun cuando basado en las manifestaciones clínicas y la recurrencia tras la reexposición se ha planteado un mecanismo de hipersensibilidad (4).

\section{Síndrome de Budd-Chiari por anticonceptivos orales (figura 8)}

Una mujer de 30 años presenta dolor y distensión abdominal. No tiene antecedentes patológicos, no toma alcohol. Toma anticonceptivos orales (ACO) desde hace 7 años. $\mathrm{Al}$ examen físico encuentran ascitis y edema de miembros inferiores, una colonoscopia refleja únicamente hemorroides internas y la ecografía muestra leve hepatomegalia, con leve agrandamiento del caudado sin estigmas de cronicidad ni masas. La resonancia nuclear magnética demuestra trombosis de la vena hepática. Las serologías virales y estudios de autoinmunidad son negativos, no hay deficiencia de proteína C o S, ni de factores de la coagulación V y II, tampoco presencia de factores procoagulantes. Las pruebas de función hepática no muestran alteraciones.

La biopsia hepática mostró importante dilatación sinusoidal con leve congestión, sin evidencia de inflamación o necrosis.

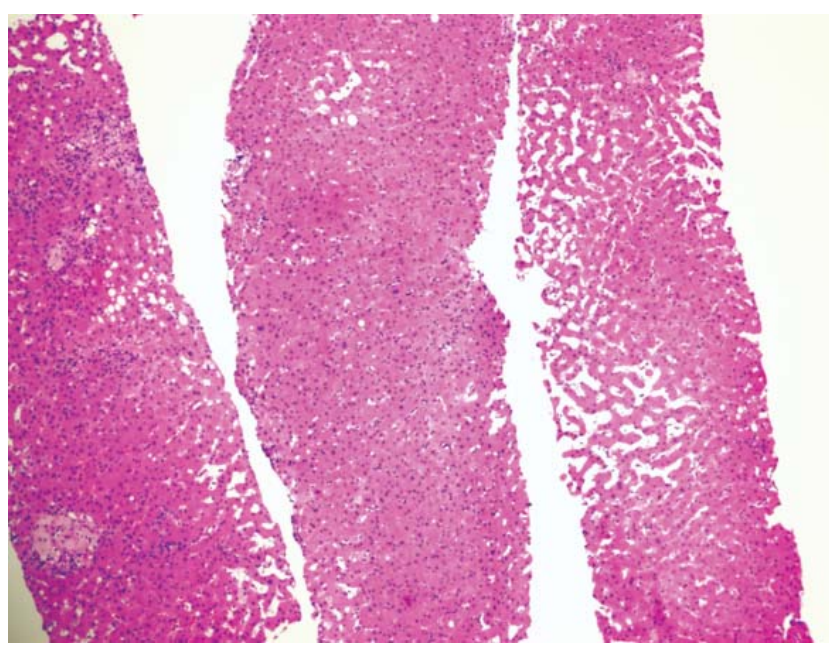

Figura 8. Hematoxilina \& Eosina 10X. Síndrome de Budd-Chiari por anticonceptivos orales. Dilatación sinusoidal pericentral.

El síndrome de Budd-Chiari y la presencia de adenomas hepáticos pueden ocurrir en mujeres con uso prolongado de anticonceptivos orales, observándose resolución de la sintomatología y regresión espontánea o marcada disminución en el tamaño tumoral luego de la suspensión de la ingesta. Para el síndrome de Budd-Chiari se ha sugerido una combinación de exposición ambiental (ACO) y predisposición genética, mientras que para los adenomas esta última no ha sido demostrada $(4,20,28)$.

\section{Fibrosis por metrotrexate (figura 9)}

Un hombre de 55 años fue diagnosticado hace 15 años de artritis reumatoidea activa, inicialmente recibió tratamiento con prednisona y penicilamina, por pobre res- 


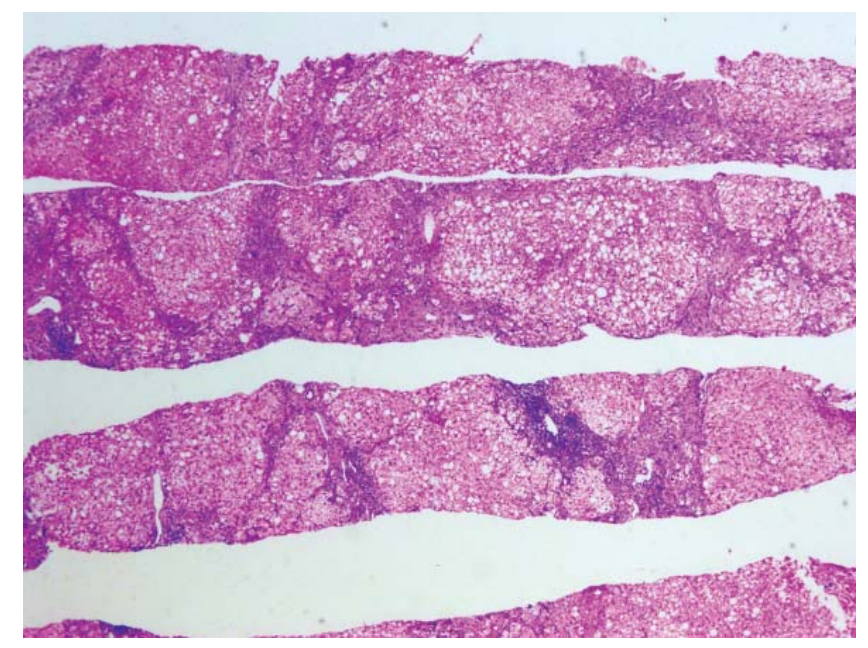

Figura 9. Hematoxilina \& Eosina. Toxicidad por metrotrexate. Alteración arquitectural, aspecto ligeramente nodular focal, por cirrosis temprana, cambio graso e inflamación portal.

puesta se inicia metrotrexate $7,5 \mathrm{mg} /$ semana y aspirina $500 \mathrm{mg} /$ día. Al inicio de la terapia las pruebas de función hepática eran normales. No hay abuso de alcohol, las serologías virales son negativas. Se aumentó progresivamente la dosis hasta $15 \mathrm{mg} / \mathrm{semana}$. Dos años después presenta un ligero aumento de las aminotransferasas (1 vez el valor normal) deciden realizar una biopsia hepática que muestra un Roenigk 1 con leve esteatosis e inflamación portal, sin fibrosis ni anisonucleosis. Continúa la terapia con mejoría notable de sus síntomas articulares, las aminotransferasas nunca tuvieron un valor mayor a 2 veces del normal. Al completar 7 años de terapia con una dosis acumulada de 4,5 gramos presenta aumento de peso y distensión abdominal, encontrando ascitis; las pruebas hepáticas mostraron leve aumento de aminotransferasas ( 2 veces valor normal), fosfatasa alcalina normal, con aumento del tiempo de protrombina a $18 \mathrm{~s}$ (valor normal $12 \mathrm{~s}$ ), albúmina $2,5 \mathrm{~g} / \mathrm{dl}$. Una nueva biopsia hepática muestra leve esteatosis y moderada inflamación con fibrosis marcada y áreas de cirrosis tem- prana (estadio 5/6), no hay cirrosis establecida correspondiendo a un Roenigk 3b. Suspenden el metrotrexate disminuyendo la ascitis y progresivamente las pruebas de función hepática, albúmina y el tiempo de protrombina fueron normales.

El metrotrexate utilizado en forma prolongada, aun en bajas dosis para tratamiento de psoriasis, artritis reumatoidea o enfermedad intestinal inflamatoria crónica. El riesgo de toxicidad hepática aumenta de forma muy importante si existe exposición a alcohol o si el paciente presenta otra enfermedad hepática de base, es obeso o diabético; en estos pacientes puede existir ya una elevación de las enzimas hepáticas que no represente de por sí un efecto toxicomedicamentoso secundario al metrotrexate, aquí la biopsia hepática puede contribuir con el manejo y la decisión médica de continuar o no la medicación.

El espectro morfológico es variado, en el análisis se tienen en cuenta la presencia de esteatosis, anisonucleosis, necrosis hepatocelular y fibrosis. La clasificación descrita por Roenigk es la más aceptada y utilizada como sistema de graduación de la lesión hepática $(29,30)$ (tabla 6).

\section{CONCLUSIÓN}

La lesión hepática inducida por fármacos, toxinas, productos herbales u homeopáticos puede imitar cualquier patrón de enfermedad primaria hepática, esto dificulta enormemente la correcta identificación de esta patología. La biopsia hepática en algunas ocasiones, se considera una herramienta muy valiosa en la evaluación de estos pacientes; por lo tanto, el informe debe ir acompañado de una detallada descripción del patrón el cual será muy útil para evaluar los posibles diagnósticos diferenciales, para determinar la severidad de la lesión y ayudar a guiar el tratamiento médico, siempre unido a un juicioso análisis clínico de una historia clínica completa y a la búsqueda de la literatura para establecer un diagnóstico correcto ya que muchas de estas sustancias comparten patrones morfológicos.

Tabla 6. Clasificación de Roenigk.

\begin{tabular}{|c|c|c|c|c|}
\hline $\begin{array}{l}\text { Roenigk } \\
\text { Grado }\end{array}$ & Cambio graso & Pleomorfismo nuclear & Necroinflamación & Fibrosis \\
\hline 1 & No hay o es leve & No hay o es leve & Con o sin leve inflamación portal & No hay \\
\hline 2 & Moderado o severo & Moderado o severo & Inflamación portal moderada o severa, necrosis lobular & No hay \\
\hline $3 a$ & Moderado o severo & Moderado o severo & Inflamación portal moderada o severa, necrosis lobular & $\begin{array}{l}\text { Leve fibrosis septal } \\
\text { e intraacinar }\end{array}$ \\
\hline $3 b$ & Leve, moderado o severo & Leve, moderado o severo & $\begin{array}{l}\text { Inflamación portal leve, moderada o severa, con necrosis } \\
\text { lobular }\end{array}$ & Moderada a severa \\
\hline 4 & Leve, moderado o severo & Leve, moderado o severo & Severo & Cirrosis \\
\hline
\end{tabular}




\section{REFERENCIAS}

1. Hou FQ, Zeng Z, Wang GQ. Hospital admissions for druginduced liver injury: clinical features, therapy, and outcomes. Cell Biochem Biophys 2012; 64: 77-83.

2. Leise MD, Poterucha JJ, Talwalkar JA. Drug induced liver injury. Mayo Clin Proc 2014; 89(1): 95-106.

3. Rangnekar AS, Fontana RJ. An update on drug induced liver injury. Minerva Gastroenterol Dietol 2011; 57(2): 213-29.

4. Ramachandran R, Kakar S. Histological patterns in druginduced liver disease. J Clin Pathol 2009; 62: 481-92.

5. Davern TJ. Drug-induced liver disease. Clin Liver Dis 2012; 16: $231-45$.

6. Kleiner DE. The pathology of drug-induced liver injury. Semin Liver Dis 2009; 29: 364-72.

7. Xuchen Zhang, Jie Ouyang, Swan N. Thung. Histopathologic Manifestations of Drug-induced Hepatotoxicity. Clin Liver Dis 2013; 17: 547-564.

8. Bjornsson E, Kalaitzakis E, Av Klinteberg V, et al. Long-term follow-up of patients with mild to moderate drug-induced liver injury. Aliment Pharmacol Ther 2007; 26: 79-85.

9. Andrade RJ, Lucena MI, Kaplowitz N, et al. Outcome of acute idiosyncratic drug-induced liver injury: long-term follow-up in a hepatotoxicity registry. Hepatology 2006; 44: 1581-8.

10. O'shea, Darasathy, and Mc Cullough Alcoholic Liver Disease. Hepatology 2010; 51(1).

11. Kleiner DE, Brunt EM. Nonalcoholic fatty liver disease: pathologic patterns and biopsy evaluation in clinical research. Semin Liver Dis 2012; 32: 3-13.

12. Rocío del Pilar López-P, y col. Ictericia colestásica inducida por metimazol en una paciente con hipertiroidismo. Acta Gastroenterol Latinoam 2014; 44: 52-58.

13. Abraham C, Hart J, Locke SM and Baker AL. A case of indometacin-induced acute hepatitis developing into chronic autoimmune hepatitis. Gastroenterology \& Hepatology 2008; 5(3): 172 - 176.

14. Czaja AJ. Drug-induced autoimmune-like hepatitis. Dig Dis Sci 2011; 56: 958-76.

15. Suzuki A, Brunt EM, Kleiner DE, et al. The use of liver biopsy evaluation in discrimination of idiopathic autoimmune hepatitis versus drug-induced liver injury. Hepatology 2011; 54: 931-9.

16. Lewis JH. Diagnosis: liver biopsy differentiates DILI from autoimmune hepatitis. Nat Rev Gastroenterol Hepatol 2011; 8: 540-2.

17. Andrew S. deLemos, David M. Foureau, Carl Jacobs, Will Ahrens, Mark W. Russo, Herbert L. Bonkovsky. Drug-
Induced Liver injury with Autoimmune Features. Semin Liver Dis 2014; 34(02): 194-20.

18. Gluck N, Fried M and Porat R. Acute Amiodarone Liver Toxicity Likely Due to Ischemic Hepatitis. IMAJ 2011; 748752.

19. Mallet VO, Bralet MP, Pol S. Response to Schiano, et al. Hepatoportal sclerosis as a cause of noncirrhotic portal hypertension in patients with HIV. Am J Gastroenterol 2008; 103: 808-9.

20. Khan AZ, Morris-Stiff G, Makuuchi M. Patterns of chemotherapy-induced hepatic injury and their implications for patients undergoing liver resection for colorectal liver metastases. J Hepatobiliary Pancreat Surg 2009; 16: 137-44.

21. Teke Z, Nessar G, Kiremitci S, Aksoy E, Elbir O. Hepar Lobatum Carcinomatosum Associated with Metastatic Rectal Carcinoma: An Unusual Cause of Liver Dysmorphy. Med Princ Pract 2011; 20(1): 93-6.

22. Gravel DH, Bégin LR, Brisson ML, Lamoureux E. Metastatic carcinoma resulting in hepar lobatum. Am J Clin Pathol 1996; 105(5): 621-7.

23. Infante PF, Petty SE, Groth DH, Markowitz G, Rosner D. Vinyl chloride propellant in hair spray and angiosarcoma of the liver among hairdressers and barbers: case reports. Int J Occup Environ Health 2009; 15(1): 36-42.

24. Hozo I Miric D, Bojic L, Giunio L, Lusic I, Culic V, Simunic M. Liver angiosarcoma and hemangiopericytoma after occupational exposure to vinyl chloride monomer. Environ Health Perspect 2000; 108(8): 793-5.

25. Dhingra S, Fiel MI. Update on the new classification of hepatic adenomas: clinical, molecular, and pathologic characteristics. Arch Pathol Lab Med 2014; 138(8): 1090-7.

26. Daniel F, Cadranel JF, Seksik P, Cazier A, Duong Van Huyen JP, Ziol M, Coutarel P, Loison P, Jian R, Marteau P. Azathioprine induced nodular regenerative hyperplasia in IBD patients. Gastroenterol Clin Biol 2005; 29(5): 600-3.

27. Bunchorntavakul C, Reddy KR. Herbal and dietary supplement hepatotoxicity. Aliment Pharmacol Ther 2013; 37: 3-17.

28. Chitturi S, Farrell GC. Corticosteroids. Adverse effects of hormones and hormone antagonists on the liver. En Kaplowitz N, DeLeve LD, eds. Drug-induced liver disease. 3rd ed. Amsterdam: Elsevier; 2013. p. 613-4.

29. GP Aithal, et al. Monitoring methotrexate-induced hepatic fibrosis in patients with psoriasis: are serial liver biopsies justified? Aliment Pharmacol Ther 2004; 19: 391-399.

30. MAM Berends, et al. Reliability of the Roenigk Classification of Liver Damage after Methotrexate Treatment for Psoriasis. Arch Dermatol 2007; 143(12): 1515-1519. 